\title{
ReMed: Jahresbericht 2015
}

ReMed, für den Leitungsausschuss: Dr. med. Peter Christen, Esther Kraft, lic. rer. oec.

\section{ReMed Schweiz - das Angebot}

ReMed ist ein Unterstützungsnetzwerk für Ärztinnen und Ärzte, welches, operativ unabhängig, von der FMH als Trägerorganisation geführt wird. Dieses Programm beinhaltet drei Handlungsfelder:

- Prävention und Gesundheitsförderung

- Unterstützung bei Krisen durch Krisenintervention, Mentoring und Coaching

- Assessments zur Überprüfung qualitativer Anforderungen.

Ziel ist es, mit gesunden und zufriedenen Ärztinnen und Ärzten eine qualitativ hochstehende und sichere medizinische Versorgung für die Bevölkerung zu gewährleisten. Dass das Dienstleistungsangebot von ReMed auch im vergangenen Jahr nötig war, zeigen die 99 Kontaktaufnahmen, welche über die Hotline oder das Kontaktformular eingegangen sind. Auffallend ist, dass sich beinahe doppelt so viele Frauen an ReMed gewendet haben als Männer. Die Problematik der Belastung am Arbeitsplatz (v.a. bei jungen Ärztinnen in der Doppelfunktion mit Mutterschaft), Burn-out sowie Depression waren die häufigsten Gründe für die Kontaktaufnahme mit ReMed. Das Durchschnittsalter der Ärzte, welche die Dienstleistungen in Anspruch genommen haben, lag bei 44 Jahren. 29 der Ärzte gaben Assistenz- oder Oberarzt als Funktion an.

\section{ReMed-Programm - Evaluation nach achtjährigem Bestehen}

Nach achtjährigem Bestehen wird ReMed durch eine externe Institution evaluiert, um so die Grundlage für Entscheidungen bezüglich der zukünftigen Ausgestaltung von ReMed zu schaffen. Ziel der Evaluation ist es, Bewährtes zu identifizieren und gegebenenfalls Optimierungspotential der Organisation von ReMed aufzuzeigen. Die gewonnenen Erkenntnisse sollen es ermöglichen, Entscheidungen bezüglich der zukünftigen Ausgestaltung des Programms ReMed zu treffen. Die Resultate werden im Frühjahr 2016 erwartet und werden alsdann umgesetzt.

\section{Starkes Netzwerk}

Ein sicheres Netzwerk von Kollegen und Kolleginnen, welche für ReMed Coaching, Beratung und Therapie von Ratsuchenden übernehmen, ist im vergangenen Jahr weiter herangewachsen. Intervisionstreffen wurden in der Deutschschweiz als auch in der Romandie erfolgreich durchgeführt. Diese laufenden Intervisionen sollen strukturiert und transparent weitergeführt werden. Für den Leitungsausschuss von ReMed ist es zentral, dass die Aktivitäten der Intervisionen auch im Rahmen des nationalen Programms abgewickelt werden. Damit diese in den Gesamtkontext von ReMed Schweiz eingebettet werden können, wird per 2016 neu ein Erfahrungsaustausch (zweimal jährlich) zwischen den Intervisionsteilnehmenden und dem Leitungsausschuss organisiert.

\section{ReMed online}

Die Website www.swiss-remed.ch wurde im Sommer 2015 überarbeitet und benutzerfreundlich gestaltet. Sie präsentiert sich neu übersichtlich, leicht und dynamisch. Nebst den wichtigen Kontaktdaten und Informationen über das Programm findet man auf der Seite auch zahlreiche Erfahrungsberichte und Dokumentationen über das Unterstützungsnetzwerk.

\section{Angebot wirkt auch präventiv}

Im 2015 bot ReMed an diversen Kongressen und Versammlungen mit Workshops und Weiterbildungen unter Einbezug von interaktivem Theater eine Möglichkeit zur Prävention und Sensibilisierung. Die Zuschauer werden jeweils durch gezielte Moderation animiert, die Handlungsweisen der Schauspielerinnen und Schauspieler nach den eigenen Vorstellungen zu gestalten. Durch das aktive Eingreifen des Publikums wird die eigene Sicht der Dinge direkt ausprobiert und die Folgen des eigenen Handelns sind unmittelbar spürbar. Diverse Testimonials, Artikel und Interviews sind im vergangenen Jahr in der Tagespresse als auch in der Schweizerischen Ärztezeitung erschienen. Diese zeigen, wie ReMed sich in verschiedenen Situationen verhalten und Hilfe angeboten hat. 